\title{
Evaluation of a color fused dual-band NVG
}

\author{
Maarten A. Hogervorst*, Alexander Toet \\ TNO Human Factors, P.O. Box 23, 3769 ZG Soesterberg, the Netherlands
}

\begin{abstract}
We have tested a prototype dual-band NVG system consisting of two NVGs fitted with filters that split the NVG sensitive range into a short (visual) and a long wavelength (NIR) band. The Color-the-night technique (see Hogervorst \& Toet, SPIE D\&S '08) was used to fuse the images of the two sensors. We designed a color scheme especially optimized for the detection of camouflaged targets. The added value of this system was evaluated in an experiment in which observers had to detect targets (green and blue tubes). Daytime images were taken with a normal camera, and NVG images were recorded at night. Performance was tested for i) the visual band only, ii) the NIR-band only, iii) normal NVG, iv) daytime imagery and v) color fused dual-band NVG. The results show that some targets were detected in the individual bands, but most targets were detected in the dual-band system, with performance comparable to that of the band optimally presenting that particular target. Our evaluation shows the added value of dual-band over single band NVG for the detection of targets, and suggests better situational awareness and perceived depth.
\end{abstract}

Keywords: image fusion, sensor fusion, human performance, false color, natural color, target detection, intensified imagery, image intensifier

\section{INTRODUCTION}

Night vision cameras are widely used for military and law enforcement applications related to surveillance, reconnaissance, intelligence gathering, and security. Most commonly used are single band cameras (e.g. Night Vision Goggles or a thermal camera) that create images with a single (one-dimensional) output per pixel. Their ability to discriminate different materials is limited. This can be improved by combining systems that are sensitive to different parts of the spectrum, such as multiband or hyperspectral imagers or systems fusing multiple sensors. The amount of different outputs can increase dramatically by combining multiple sensors (e.g. when the number of different outputs for each sensor is $N$, this can increase by up to $N^{2}$ for two sensors). This also leads to a dramatic increase in the number of materials that can be discriminated. The combination of multiple bands allows for presentation of the output in color in a meaningful way. It is therefore not surprising that the increasing availability of fused and multiband infrared and visual nightvision systems has led to a growing interest in the color display of night vision imagery ${ }^{1-5}$. In principle, color imagery has several benefits over monochrome imagery for surveillance, reconnaissance, and security applications. Color may improve feature contrast, allowing better scene recognition and object detection ${ }^{6}$. Color can be used to discriminate between different materials (e.g. in X-Ray baggage screening the sensor output at two energies for material classification). However, the coloring method should be chosen with care, since unnatural coloring may disrupt the recognition process, resulting in observer performance that is even worse compared to with single band imagery ${ }^{7}$. Recently, we presented a method for applying natural daytime colors to multiband nightvision imagery ${ }^{8,9}$. Our method is simple and fast, can easily be deployed in realtime, and can display night-time imagery in natural daytime colors, that are stable under variations in scene content. Different color schemes can be used, depending on the task at hand. For our application we devised a color scheme that is particularly suited for the detection of targets. We created a prototype dualband Night Vision Goggle (NVG) system consisting of two optically aligned NVGs fitted with filters. One filter transmits the visual part (short wavelength region), the other the near infrared part (long wavelength region) of the NVGsensitive range. The images of the two sensors are combined and color is added using the Color-the-night method. Here, we present the results of a human observer experiment to establish the added value of such a system (over standard NVG or each of the individual bands) for the detection of targets.

* maarten.hogervorst@tno.nl; phone +31-346-356241; fax +31-346-353977

Multisensor, Multisource Information Fusion: Architectures, Algorithms, and Applications 2009, edited by Belur V. Dasarathy, Proc. of SPIE Vol. 7345, 734502 - (c) 2009 SPIE · CCC code: 0277-786X/09/\$18 · doi: 10.1117/12.819114 


\section{COLOR FUSION METHOD}

The color schemes that are suited for most applications produce colors that i) closely match the (natural) daytime colors and ii) lead to good discrimination of different materials. Previously we reported on ways to create a natural color scheme $^{8}$. These two requirements are not always in line and may be contrasting. A typical example of this (as we have found) is presented by the task of finding a camouflaged soldier in a field by using a thermal sensor and NVG. When the colors are chosen such that the match with the daytime colors is optimized, this will result in a soldier that is camouflaged. This is obviously undesirable. In such cases more emphasis should be placed on the second requirement, i.e. using a color scheme that leads to better discrimination but also less natural colors. Also for our purpose of optimal detection of targets we found that the use of natural colors does not lead to optimal performance. We therefore designed a color scheme that is targeted at good detection of (camouflaged) targets that do not contain chlorophyll, and leads to reasonably natural colors.
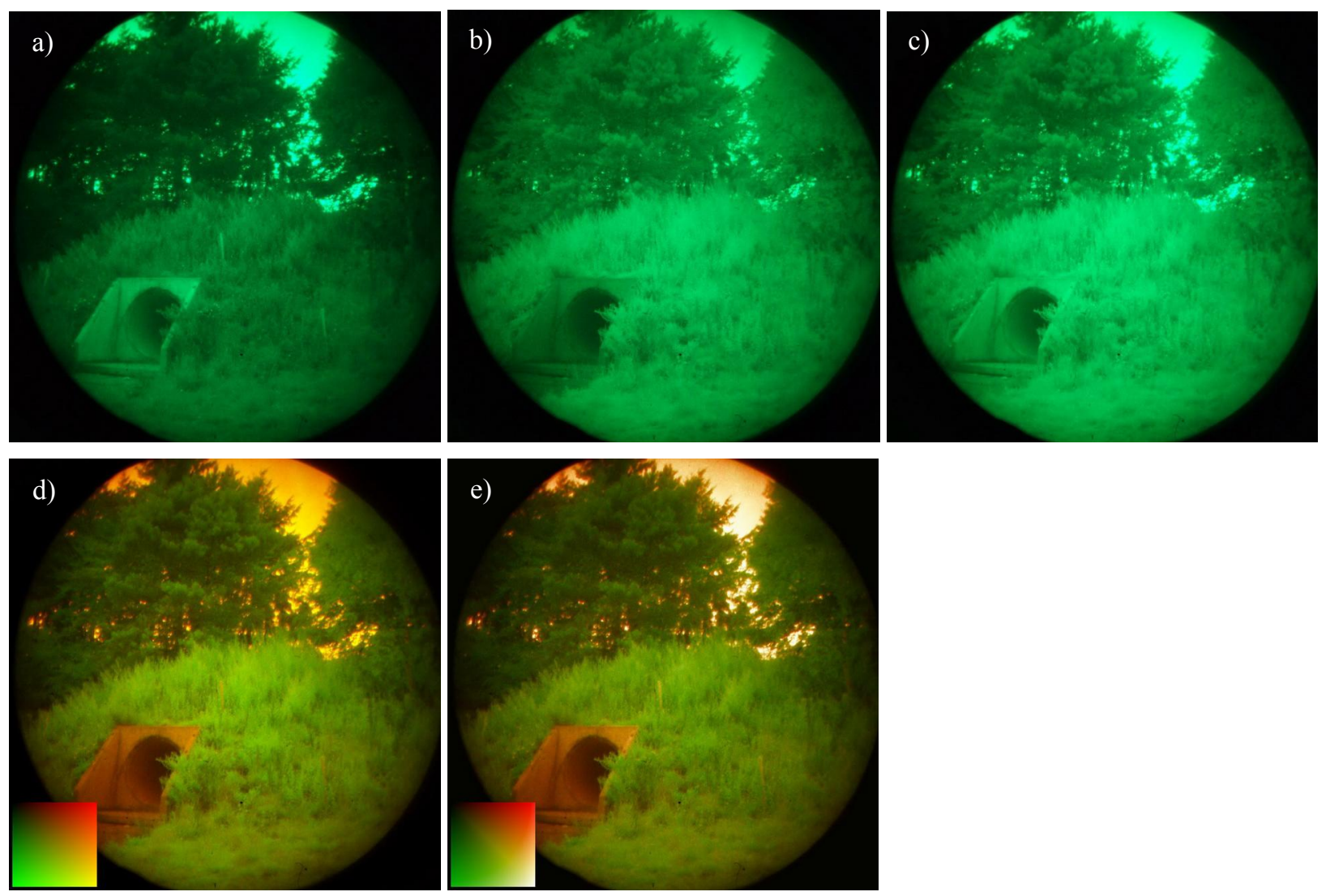

Figure 1. Example showing our color fusion method applied to a dual-band NVG sensor consisting of a NVG fitted with a filter transmitting wavelengths lower than $700 \mathrm{~nm}$ (Figure 1a), and an NVG sensor with filter transmitting wavelengths higher than $700 \mathrm{~nm}$ (Figure 1b). Figure 1d shows a representation of the dual-band image with the short wavelength band in Red and the long wavelength band in Green. The inset in Figure 1d shows all possible dual-band outputs as shades of red (large response in band 1, small in band 2), green (small response in band 1, large in band 2) and yellow (large responses in both bands). Figure 1e shows the result of the color transformation. The inset shows how the colors in the inset of Figure 1d are transformed. Figure 1c shows the image of a standard (single band) NVG for comparison.

An optically aligned dual-band NVG sensor was simulated by using a NVG and recording images (with the use of a standard digital daytime camera) with various filters in front of the NVG. A "visual band" band was created by using a filter transmitting wavelengths shorter than $700 \mathrm{~nm}$ (Figure 1a). A "near infrared" (NIR) band was created by using a filter transmitting wavelengths longer than $700 \mathrm{~nm}$ (Figure 1b). For comparison we also created a standard NVG image of each scene without the use of any filters (Figure 1c). The visual band was fed into the Red channel of an RGB-image and the NIR-band was fed into the Green channel, to create a red-green representation of the dual-band sensor image (Figure 1d). Next, for each combination of sensor outputs (represented by a shade of red, green, yellow; see inset of 
Figure 1d) a color was chosen to display this sensor output. This process can be implemented by transforming the redgreen image (Figure 1d) into an indexed image in which each pixel value refers to the entry of a color lookup table. When a color lookup table is used with different colors, the colors in the indexed image are automatically transformed into other colors, in a way that all pixels with the same index will result in the same color. Details of this can be found in Hogervorst \& Toet $^{8}$. We tried several color transformations in our search for a color scheme that results in optimal detection of targets as well as natural looking images. The best color transformation we found for our purposes looks similar to the red-green representation with a few changes. The inset of Figure 1e shows the colors attributed to all dualband outputs (represented by the inset of Figure 1d) of the chosen color scheme. This color scheme emphasizes the distinction between objects containing chlorophyll (the background plants) and objects containing no chlorophyll (e.g. our targets; notable from the sharp transition between green and red at the diagonal). To create a more natural look objects that lead to high response in both channels are depicted in white (bottom right corner of the inset of Figure 1e). The result of our color fusion method is shown in Figure 1e.

\section{EVALUATION METHOD}

We evaluated our color fusion method using a target detection task. We recorded images with and without targets during daytime (without NVG) and nighttime (with NVG) in the same area containing grass and trees (see Figure 2). Performance for detecting targets was established for imagery of the dual-band fusion system, each of the individual NVG-bands (visual and NIR), standard NVG and daytime images (taken with a visual camera). The daytime images were matched to the NVG-images in Field of View (visual angle and display area), by using a circular mask and using the same zoom with and without NVG (see Figure 2).

The conditions for which performance was measured are:

a) Daytime: taken with a standard digital daytime camera

b) $\quad N V G$ : standard NVG, without filter

c) NVG-visual: NVG with filter transmitting short wavelengths

d) NVG-nir: NVG with filter transmitting long wavelengths

e) NVG-dual band: images resulting from the color fusion method

The targets were green (Figure 2a) or blue (Figure 2b) foam tubes. The reflectance of the tubes was such the green tubes were often undetectable in the standard NVG and the near infrared band (see Figure 1), but detectable (as a light object) in the visible band (see Figure 1). In contrast, the blue tubes were often undetectable in the visual band while being detectable (as a dark object) in the near infrared band and in standard NVG (see Figure 3).
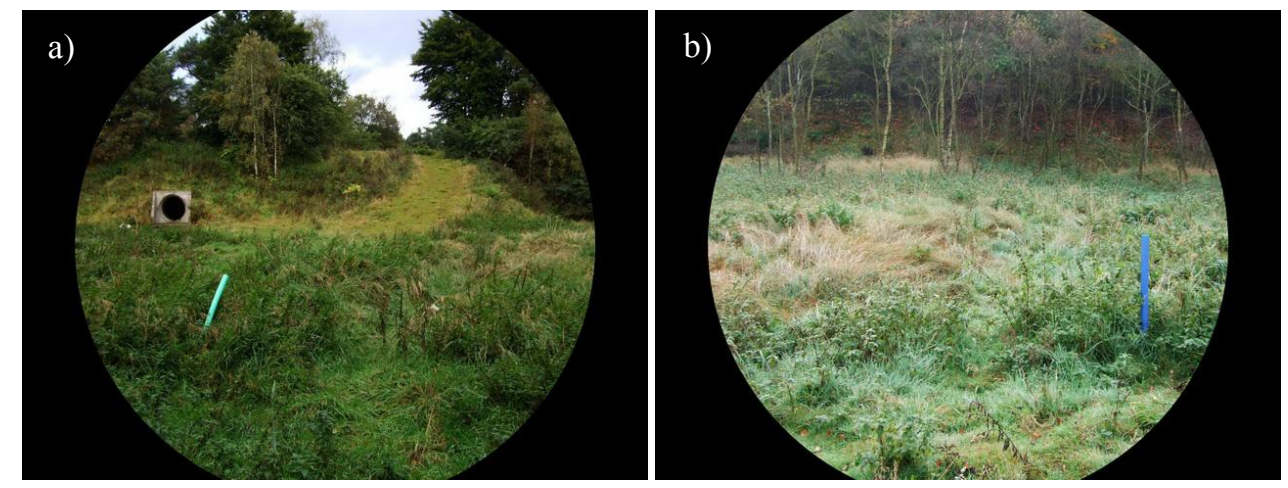

Figure 2. Images showing the two target types, the green target (Figure 2a) and the blue target (Figure 2b). 

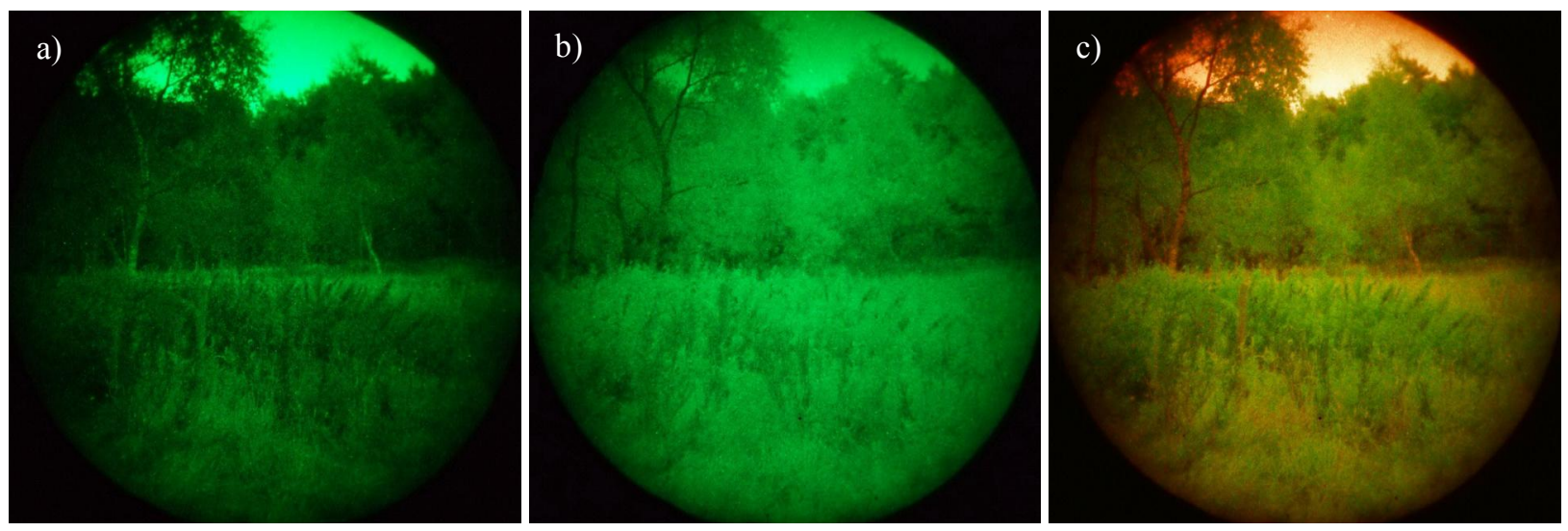

Figure 3. Example showing the visual band (Figure 3a), near-infrared band (Figure 3b) and the color fused dual-band image

(Figure 3c) for a scene that includes a blue target. The target is visible in the near-infrared band as a dark tube. The dual-band image shows the target as a reddish tube.

In each condition 56 images were used without target, 26 with a green target and 26 with a blue target. Seven subjects participated in the experiment. Each subject participated in 5 sessions in which the stimuli of each condition were shown separately. Each subject started the session with the Daytime condition to get acquainted with the procedure. The order of the NVG-conditions was randomized across subjects to compensate for possible training effects. The images were shown on a PC monitor with a resolution of 1600x1200 pixels (Figure 2 gives a realistic view of the display content; Figure 1 does not!).

Each experimental session started by explaining the purpose of the experiment and by showing some example stimuli of each condition. Each trial started by showing an image. The subject was required to decide as quickly as possible whether a target was present or not. As soon as this decision was taken he/she clicked the mouse button. Next, the image disappeared and was replaced by a low resolution equivalent of the image, consisting of 20x15 uniformly colored squares (to prevent subjects from searching for the target after responding). We registered the time between onset of the stimulus and detection (the response time). The subject then indicated the target location or clicked on an area outside the image labeled "no target found". When the subject did not respond within 8 seconds the trial was ended automatically. The indicated target location was used to check whether the subject had detected the target or had found a false target (responses outside an ellipse with horizontal diameter of 162 and vertical diameter of 386 pixels centered round the (vertically elongated) target were treated as incorrect). 


\section{RESULTS}

We recorded whether the subjects detected the targets when present (Hits and Misses) and whether they judged there to be a target when no target was present (False Alarms and Correct Rejections). We also recorded the response times. No False Alarms were recorded, i.e. the False-Alarm rate was zero. Performance is therefore fully characterized by the Hitrate, i.e. the fraction of targets that was detected $\left(p_{h}=\#\right.$ Hits / (\#Hits $+\#$ Misses $)$ ).

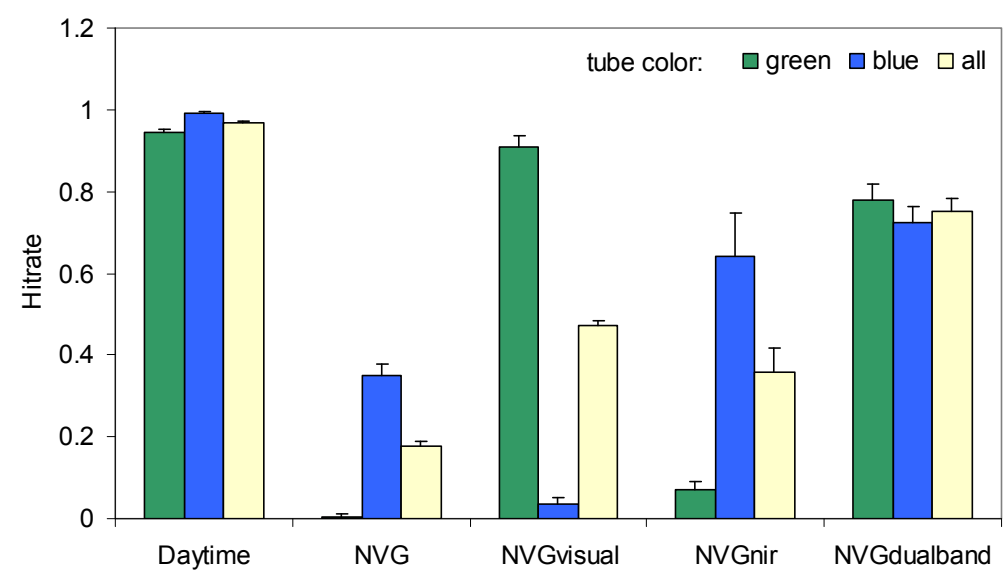

Figure 4. Average hit-rate (fraction of hits) for the various sensor conditions and target colors, including the hit-rate for all target colors ("all"). Shown are averages over all subjects. The error bars are standard errors in the mean derived from the variance between subjects.

Figure 4 shows the fraction of hits (hit-rate) for the various sensor conditions and target colors. Shown are the average hit-rates over subjects. Not surprisingly, performance is highest in the Daytime condition. As expected (see Figures 1 and 3 ), performance for detecting the green targets is high in the NVGvisual condition and low in the NVG and NVGnir sensor conditions. Performance for detecting the blue targets is somewhat poorer in the single-band conditions. These targets can be detected in the NVGnir condition (reasonably well) and in the $N V G$ condition (poorly), while they are hardly detected in the NVGvisual condition. Detection performance for both targets is high with the dual-band sensor. Optimal fusion results in performance that equals maximum performance in the individual bands. The hit-rate for the green targets is somewhat lower for NVGdual-band than for NVGvisual. But the hit-rate for the blue targets is somewhat higher for NVGdual-band than for NVGnir. The average hit-rate of the NVGdual band sensor (0.75) is not significantly different from the average of the hit-rate for green in NVGvisual and the hit-rate for blue in NVGnir (0.78). This means that this fusion scheme is (close to) optimal. The results also show that the performance with the standard NVG is clearly much worse than with the dual-band NVG system.

a)

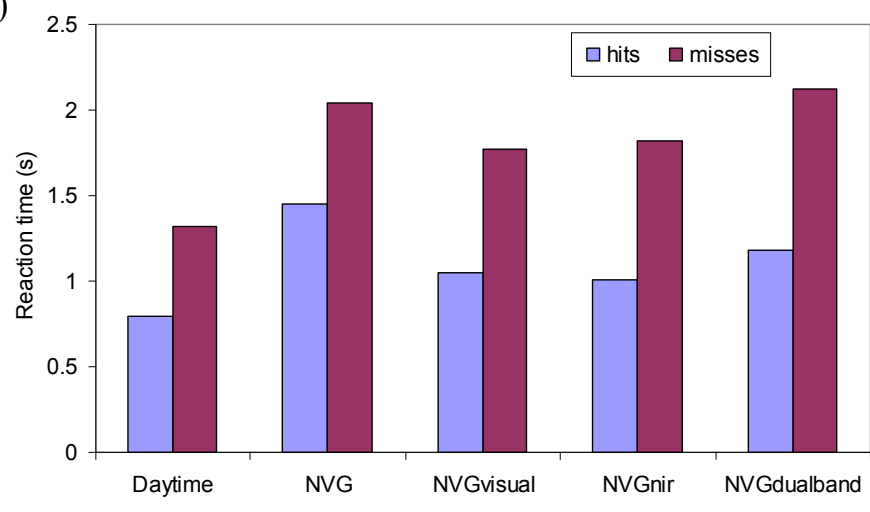

b)

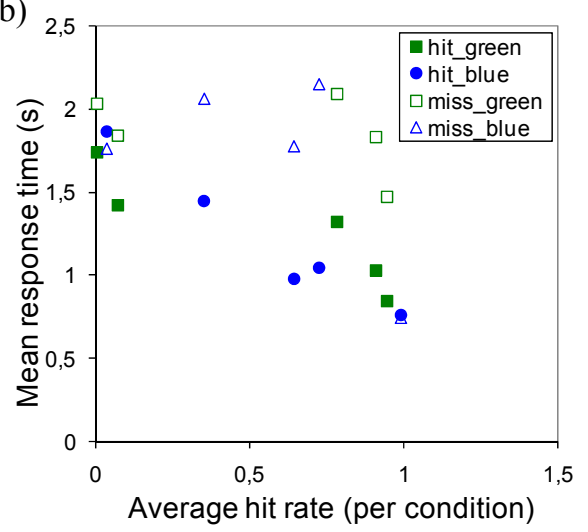

Figure 5. Figure 5a shows the geometric mean (i.e. averaged in log) response times for the various sensor conditions separated for hits and misses. Figure $5 \mathrm{~b}$ shows the relationship between the hit-rate for each sensor condition and the (geometric) mean response times for hits and misses for the two target colors. 
Figure 5a shows the response times of the trials containing a target (shown are the geometric means over the response times, i.e. the exponent of the average log response times) for all conditions for the hits and misses. Note that the hits in NVGnir and NVG correspond primarily to the trials containing blue targets; the hits in NVGvisual correspond primarily to the trials containing green targets. The response times in the dual-band condition are comparable, but somewhat larger than in the single-band NVG conditions with filter. This may be due to the fact that in this condition subjects had to attend to two types of targets, while in the single band conditions only one of the target colors was apparent.

It turns out that the response times for missed targets is comparable to the response times for stimuli in which no target is present. The average response times for missed targets in the conditions do not correlate with the hit-rates (see Figure $5 b)$. In contrast, the average response times for hits in the conditions is highly correlated with the hit-rate $(r=-0.90, p<$ 0.01 , see Figure $5 \mathrm{~b}$ ). This indicates that when targets are more easily detected, the hit-rate goes up and the response time goes down.

\section{CONCLUSIONS}

We have designed and evaluated a dual-band NVG system. In previous studies ${ }^{8,9}$ a color fusion method was used that results in an image with colors that closely match the natural daytime colors. Although such color schemes may be optimal for other tasks such as creating situational awareness, this scheme does not appear to be optimal for target detection. We designed a color scheme that is optimized for detecting (our type of) targets. Although the scheme is not optimized for matching the daytime colors, the color scheme results in colors that are intuitively in line with the daytime perception of the scene. The color scheme emphasizes the distinction between plants (containing chlorophyll) and nonplants (not containing chlorophyll). The sensor system separates the NVG sensitive region in a part with wavelength below $700 \mathrm{~nm}$ and one with wavelengths above $700 \mathrm{~nm}$. Since chlorophyll shows a steep rise around 700nm, this dualband NVG system is especially suited for discriminating materials containing chlorophyll from materials containing no chlorophyll. Elements containing chlorophyll (e.g. plants) are displayed in green (i.e. in their natural color), while objects without chlorophyll are displayed in the perceptually opposite color red. To further increase the naturalness, elements with high output in both channels are displayed in white.

This prototype dual-band color fused NVG system was evaluated in a human observer experiment in which performance for detecting green and blue tubes was established. The results clearly show that performance for detecting these targets is much better with the dual-band NVG system than with standard NVG. Moreover, the results show that performance of the dual-band system is as good as the maximum performance of both individual bands. While the green targets could well be detected with the visual band of the system alone, the blue targets were largely missed when subjects had to rely on this band alone. In contrast, the blue targets could well be detected with the near-infrared band alone, but the green targets were then largely missed. With the dual-band sensor both targets could be detected. The total number of targets that was detected with the dual band system was the same as the total number of targets that was detected with the visual band plus that was detected with the near-infrared band. This indicates that the way the fusion of the two bands is implemented is (near) optimal.

The above finding suggests that one can as well present the two bands side by side. For such a display performance can be expected to be comparable to that of the fused image. However, longer response times are expected for this alternative presentation. Also, in some situations one may encounter targets that cannot be detected in either of the individual bands. This may be the case when one part of an object is visible in one band and the other part in the other. Likewise, when several elements are available that are present in the different bands, the spatial relationship between the elements is difficult to judge. Also other advantages of the sensor fusion are lost, including better identification of object and materials, better situational awareness and better depth perception (e.g. compare Figure 1e with 1c). Indeed, informal accounts of the subjects suggest that the color fused images give a better sense of depth and are judged to be easier to interpret.

The simulated prototype sensor system is highly similar to the prototype system called the Gecko we build and presented earlier ${ }^{8}$, which consists of two optically aligned NVGs fitted with similar filters as used here. Our experiences with this dual-band NVG sensor system show that our color transformation method can be implemented in realtime. Another prototype we developed is called the Viper'. The Viper provides co-aligned images from a digital image intensifier and an uncooled longwave infrared microbolometer. Since the sensitive range of the dual-band NVG system is close to the visual range, the output values are well correlated with the daytime colors. Therefore, this system is suited for presenting the image in natural colors. The image of a thermal sensor is very different from the daytime image, and the output is 
often poorly correlated with the daytime color. However, a thermal image contains different information that can be highly useful, for instance for detecting and locating (hot) targets. We are currently investigating ways to combine a dual-band NVG system with a thermal imager ${ }^{10}$. We expect that the dual-band NVG can be used to create natural looking backgrounds, while the thermal imager can be used to highlight potential (hot) targets.

\section{REFERENCES}

1. Li, G. and Wang, K., Applying daytime colors to nighttime imagery with an efficient color transfer method, In: J.G. Verly \& J.J. Guell (Ed.), Enhanced and Synthetic Vision 2007, pp. 65590L-1-65590L-12, The International Society for Optical Engineering, Bellingham, MA, 2007.

2. Shi, J., Jin, W., Wang, L. and Chen, H., Objective evaluation of color fusion of visual and IR imagery by measuring image contrast, In: H. Gong, Y. Cai \& J.-P. Chatard (Ed.), Infrared Components and Their Applications, pp. 594601, The International Society for Optical Engineering, Bellingham, MA, 2005.

3. Shi, J.-S., Jin, W.-Q. and Wang, L.-X., Study on perceptual evaluation of fused image quality for color night vision, Journal of Infrared and Millimeter Waves, 24(3),pp. 236-240, 2005.

4. Tsagaris, V. and Anastasopoulos, D., Multispectral image fusion for improved RGB representation based on perceptual attributes, International Journal of Remote Sensing, 26(15),pp. 3241-3254, 2006.

5. Zheng, Y., Hansen, B.C., Haun, A.M. and Essock, E.A., Coloring night-vision imagery with statistical properties of natural colors by using image segmentation and histogram matching, In: R. Eschbach \& G.G. Marcu (Ed.), Color imaging X: processing, hardcopy and applications, pp. 107-117, The International Society for Optical Engineering, Bellingham, WA, 2005.

6. Walls, G.L. (2006). The vertebrate eye and its adaptive radiation. Bloomfield Hills, Michigan: Cranbrook Institute of Science.

7. Sinai, M.J., McCarley, J.S. and Krebs, W.K., Scene recognition with infra-red, low-light, and sensor fused imagery, In: Proceedings of the IRIS Specialty Groups on Passive Sensors, pp. 1-9, IRIS, Monterey, CA, 1999.

8. Hogervorst, M.A. and Toet, A., Nighttime imagery in natural daytime colors, In: B.V. Dasarathy (Ed.), Multisensor, Multisource Information Fusion: Architectures, Algorithms, and Applications 2008. The International Society for Optical Engineering, Bellingham, WA, USA, 2008.

9. Toet, A. and Hogervorst, M.A., Portable real-time color night vision, In: B.V. Dasarathy (Ed.), Multisensor, Multisource Information Fusion: Architectures, Algorithms, and Applications 2008, The International Society for Optical Engineering, Bellingham, WA, USA, 2008.

10. Toet, A. and Hogervorst, M.A., The TRICLOBS portable triband lowlight color observation system, In: B.V. Dasarathy (Ed.), Multisensor, Multisource Information Fusion: Architectures, Algorithms, and Applications 2009, The International Society for Optical Engineering, Bellingham, WA, USA, 2009. 